\title{
Artificial Intelligence in Mineral Processing Plants: An Overview
}

\author{
Luis Bergh* \\ Chemical Engineering Department, Santa Maria University, Valparaiso, Chile \\ ${ }^{*}$ Corresponding author
}

\begin{abstract}
This work review the applications of artificial intelligence in the field of mineral processing in the last decade. The mineral processing industry is characterized for the lack of reliable models and on-line information about key process variables. Therefore, heuristic methods have been extensively used to accommodate the plant operation looking for better metallurgical results. Applications to flotation plants are discussed.
\end{abstract}

Keywords-artificial intelligence; applications; mineral processing; flotation

\section{INTRODUCTION}

The aim is to concentrate a raw ore in a technically coherent sequence of processes for the subsequent metal extraction stage. Usually, the valuable minerals are first liberated from the ore matrix by comminution and size separation processes, and then separated from the gangue using processes capable of selecting the particles according to their physical or chemical properties [1]

The objective of this contribution is to make a survey of the progresses and challenges in the field of artificial intelligence of flotation processes. A more complete discussion of the general state of the art and challenges in mineral processing can be found in $[1,2]$. Although the presentation scope is quite large, only some key references are proposed for further details.

\section{A. Flotation Process}

Froth flotation processes have been over a century in the mineral processing industry. However, despite numerous years of research and development, flotation is still not fully understood and remains relatively inefficient, opening opportunities to novel methodologies for its optimization.

Information about the input disturbances, process operating parameters and final product quality is required before optimization and control can be performed. As it is well known, the quality of measured information largely determines the efficiency of any implemented control system. Instrumentation for the measurement of important parameters such as: ore composition, flow rates and some ore specific properties (e.g. density, pH, pulp levels, particle size) have been available, however, some essential properties such as liberation degree, surface chemistry, bubble size distribution, bubble loading remain difficult to measure and infer $[2,3]$.

\section{B. Modeling and Control Difficulties}

The processes are inherently stochastic and difficult to model, and the measurements quite inaccurate, while the most important variables are not measurable on-line.

A large number of different variables are important in flotation process, such as: feed characteristics (mass flow rate, mineral composition, liberation size, particle size distribution, specific gravity, etc.), physicochemical factors (water quality, temperature, reagent types and concentrations, interactions between reagents and particles, etc.) and hydrodynamics (flotation circuit design, cell type, aeration rate, spatial distribution of bubbles and particles, etc.). Process mechanics and dynamics are very complex and it is vastly difficult to take reliable, accurate or direct measurements of certain process variables. Therefore, reliable models for simulation or control of flotation processes are hard to obtain [4].

Distributed control systems of local objectives (e.g. pulp level, flow rates) are not sufficient to accomplish appropriate criteria for concentrate grade, therefore supervisory control systems with different attributes are needed. These systems should be adaptable to different computation platforms and should at least consider modules for: validation and reconciliation of process data, detection of operation and instrumentation problems and co-ordination of local control loops under an overall strategy [5].

Despite much research into both empirical and phenomenological based modeling, several issues remain. Multivariate predictive control is ideally the solution for high quality control. However, to be applicable without losing its benefits, good measurements, acceptable regulatory control of local objectives, reliable dynamic models, explicitly stated process constraints and new methods to promote robustness are needed $[3,6]$. Flotation processes have weaknesses in most of those aspects.

Furthermore, design of control systems are insufficiently thought out during plant design stages and major control variables are often only identified once the plant is operational [7]. This may result in poorly designed ad-hoc control systems. Even more, a majority of operators, metallurgists and management do not have a background in control engineering, making more difficult to model and control non-linear, complex processes, in large ranges of operating conditions $[2,5$, 7]. 
Some critical aspects of control strategies are [3]: measurement instrumentation, data reconciliation, pattern recognition, fault detection and diagnosis, soft sensors, process and controller performance monitoring. The successful utilization of knowledge-based control strategies related to flotation is mostly dependent on the quality of information and process knowledge.

\section{ARTIFICIAL INTELLIGENT APPLICATIONS}

Soft computing is a collection of techniques in the area of artificial intelligence, which exploit the tolerance for imprecision, uncertainty and partial truth to achieve tractability, robustness and low solution cost. Soft computing methodologies and tools can assist in the design, development and operation of intelligent systems that are capable of adaptation, learning and operating autonomously [8].

These techniques allow the model and control designer to take advantage of the knowledge accumulated, either in a linguistic form or in a form of other data, to continually learn from the operational experience and to utilize the possibilities of intelligent algorithms for process optimization [9].

The most commonly used artificial intelligence methods in flotation plants are: fuzzy logic, artificial neural networks, genetic algorithms, support vector machines, decision trees and hybrids of these methods [8].

\section{A. Application of Artificial Neural Networks}

Several authors have reach the conclusion that ANN models are superior in relation to statistical methods especially when applied to copper sulfide flotation concentration process. ANN modeling has also applied to describe the dependency of technological parameters of copper flotation process on the various process parameters, such as copper feed content, reagents dosage and feed size distribution [8].

ANN is used extensively within the areas of identification, categorization and interpretation of flotation froth images, within the computer vision systems [10]. One of the possibilities for neural network application within this area is the extraction of froth images features. However, neural networks with multiple hidden layers can be difficult to train and may not yield consistent or robust results [10]. Other application is found in the estimation of mean bubble diameter and bubble size distribution on the mineralized froth surface.

\section{B. Application of Fuzzy Logic}

Fuzzy logic is considered a suitable instrument for modeling complex and poorly-defined systems, since present capabilities to efficiently approximate reasoning rather than exact reasoning [8].

Fuzzy models have been used to characterize certain subsystems within flotation systems, such as: the pulp level, and air holdup. However, there are fuzzy logic models that can interpret the flotation processes as a whole, through the contents and recovery of valuable component in the concentrate and tailings.
There are several fuzzy controllers already operating in the flotation plant, particularly for flotation columns. The main reason for this is the operational flexibility that exhibit flotation columns when compared to mechanical cell circuits. There are more variables available to shape its operation to the feed characteristics to meet the metallurgical targets $[3,5]$. The level of detail with which some fuzzy models are developed, is reflective of a more developed approach. This is probably the consequence of easier, more comprehensive and approachable fuzzy logic tools, in comparison with ANN development tools [8].

\section{Application of Genetic Algorithms}

Genetic algorithms have the ability to initiate the search for an optimal solution from the series of possible solutions that represent a starting population of genetic algorithm.

In the field of flotation system modeling, genetic algorithms are most commonly used for the optimization of flotation circuit configuration, competing with the expertise of practitioners [8]. However, it can be concluded that the majority of optimal solutions obtained by genetic algorithms only carry theoretical importance, since some practical aspects are not well defined. In practice, process complexity reduces the possibility of potential control and flotation process regulation.

\section{Application of Support Vector Machine}

Support vector machine (SVM) is a concept in machine learning, for a set of related supervised learning methods, that analyze data and recognize patterns used for classification and regression analysis [8].

SVM methods in flotation processes modeling are being increasingly accepted, however, the regression method is currently much more utilized than the classification method. The SVR is used in the prediction of concentrate grade and recovery, based on the features extracted from images of flotation froth. This method is often used in combination with other AI methods [8].

\section{E. Application of Decision Trees}

Decision trees are used in a form of predictive models which, based on the data collected about a specific problem, produce conclusions about the solution of the problem in question. There are only a few reports regarding the modeling of the flotation system by use of the inductive decision tree methods [8].

Regardless of the advantages with the application of decision trees in the modeling of flotation processes, some authors [10] have suggested the use of contemporary techniques such as the random forests method.

\section{F. Other Soft Computing Methods}

Other techniques can also be applied in the optimization of flotation models parameters, such as: particle swarm optimization, glowworm swarm optimization, gravitational search algorithm, differential evolution, artificial immune systems and cuckoo searching algorithm [8]. 


\section{G. The Hybrid (Combined) Approach}

Some authors have suggested applying the hybrid approach to flotation system modeling, with the aim of providing a more accurate description of the flotation process performances. Hybrid modeling can encompass some of the soft computing methods, or a combination of those with classic mathematical modeling.

For example, a fuzzy inference system as a part of the control strategy that was aimed to optimize the copper flotation process in a flotation column was discussed in [11]. Fuzzy model was structured through three logic tables (i.e. three sets of rules), where the errors in recovery and the concentrate grade were considered as the inputs, and the outputs were the desired froth depth, air flow rate and wash water flow rate. The authors concluded that the fuzzy system had provided smooth process dynamic responses (especially when constraints were imposed). The fuzzy system however required tuning of more parameters than the binary logic system and was consequentially, more time consuming in the initial stage when it was under supervised control. A hybrid approach reduces the dimensionality of the problem by combining expert and fuzzy systems.

\section{DISCUSSION}

Some aspects discussed here have been taken from the experience of many authors, among them $[2,3,4,7,8,9,10$ and 12]. The discussion is organized in three sections regarding the constraints imposed by the process, those inherent to the artificial intelligent methods and finally a summary of the actual criticisms of practitioners and future expectations.

\section{A. On the Process Constraints}

The complexity of the flotation process, as well as the availability of quality industrial data and expert knowledge in flotation and soft computing have largely dictated the model development conditions.

The multidimensional complexity of the mineral raw material and the flotation process explains why artificial intelligence methods are often considered suitable for interpretation and optimization of flotation systems.

The stochastic nature of disturbances coming into a flotation process poses mathematical modeling and control problems difficult to standardize or look for a global solution. Usually, plant differences require adaptation of solutions according to its nature and constraints.

Even when the mineral raw material properties were completely known, it still does not guarantee optimal process performance. In any case, a control system will have only limited capabilities for controlling the whole flotation system, since some phenomena such as, attachment, detachment, and entrainment, will always occur with a significant degree of unpredictability. On the other hand, all resources, that can be used to accommodate the operation of a unit, can become saturated as well.

In flotation plants the first target will be to adjust the operating conditions looking for a solution that is close enough to optimal. This will be satisfactory accordingly to the actual process knowledge and constraints.

\section{B. On the AI Constraints}

A common characteristic of these applied ideas is that all of them are unique and can be implemented on an individual basis, and thus, have not yet been developed into a more generalized approach, or general model of the flotation process. It is evident that the application of artificial intelligence methods in flotation systems research is still in its preliminary phase [8].

For control and management people in flotation plants, the application of fuzzy logic and neural networks can be aid for commercial software packages. Other artificial intelligence methods are less understood and therefore also the adequacy of application with the method properties.

All the methods presented here are considered suitable enough to be the basis of an intelligent system, but the methods used are very different when we consider their characteristics. In the cases discussed in the literature, there is an attempt to cover various sorts of problems that researchers and engineers encounter on a daily basis, with the same tools.

From experience, it is difficult to reason that different artificial intelligence methods can cover or solve all of the same problems. It is more logical to assume that the answer to the optimal application of these methods lies within a certain combination or even synthesis of these methods leading to hybrid approaches.

The true hybrid approach would mean that the artificial intelligence methods are mutually supplementing each other, but there have only been a few examples of this in practice. The main reason for this could be the lack of knowledge of potential outcomes and results. A hybrid approach may improve the results in flotation process optimization.

\section{Today Criticism}

Artificial neural networks have shown effective results in in predicting process parameters of flotation processes. The results obtained by the use of a single method are often better in comparison to other regression methods. Fuzzy logic models are also considered to be an applicable tool for the creation of expert systems. It can be reasoned that both genetic algorithms and decision trees are still yet to be developed in a more practical sense. It seems, however, that the hybrid types are the most promising and reliable soft computing methods thus far.

The majority of published papers have been written on the topics of improvements, good or positive results, and even the successes in flotation process modeling. Some important facts, however, should be taken into account [8]:

- Many studies include only laboratory or pilot plant tests, with only a limited number of experiments, some of which are developed only on the grounds of a two-phase system (without the solid phase that basically dictates the flotation process), or purely on theoretical considerations.

- Many studies shown advantages of artificial intelligence methods over more conventional and known methods based on 
idealized simulations (not including all constraints and variety of real problems).

- Industrial data-based research is commonly taken into account only for a short period of time during plant operation, and it is therefore arguable as to whether the same model is able to describe the process as effectively when applied for a longer period of time.

- Nearly all the research is related only to a single stage of flotation (the most common is rougher flotation), therefore it completely disregards the events in other flotation stages, which are certainly still important to the characterization of the flotation process as a whole.

In particular in froth image attributes interpretation there are some issues to consider [2010]:

- It is commonly observed that the surface bubbles of flowing froths are significantly larger than those in the layers immediately below; the lower layers forming the predominant portion of the volume overflowing into the launder. This cannot be corrected for readily.

- The surface film size distribution is not necessarily representative of the bubble size distribution in the underlying froth layer.

- The watershed method often over-segments larger bubbles and under-segments smaller bubble sizes.

In general, unfortunately these techniques are quite complex and the proposed modeling and control architecture is so difficult to be implemented in a robust form.

\section{ACKNOWLEDGMENT}

The author would like to thank the financial support from UTFSM Project 678717 and Fondecyt Project 11300568.

\section{REFERENCES}

[1] D. Hodouin, Jämsä-Jounela, S.-L., Carvalho, M.T., Bergh, L., 2001 State of the art and challenges in mineral processing control. Control Engineering Practise 9, 995-1005.

[2] D. Hodouin, 2011, Methods for automatic control, observation, and optimization in mineral processing plants, Journal of Process Control, Volume 21, Issue 2, February 2011, Pages 211-225

[3] Bergh, L.G., Yianatos, J.B., 2011. The long way toward multivariate predictive control of flotation processes. Journal of Process Control 21, 226-234.

[4] Laurila, H., Karesvuori, J., Tiili, O., 2002. Strategies for instrumentation and control of flotation circuits. Mineral Processing Plant Design, Practise and Control, Volume 1, pp. 2174-2195. ISBN-10: 0873352238.

[5] Bergh, L.G., Yianatos, J.B., Acuña, C.A., Pérez, H., López, F., 1999 Supervisory control at Salvador flotation columns. Minerals Engineering 12 (7), 733-744

[6] McKee, D.J., 1991. Automatic flotation control - a review of 20 years of effort. Minerals Engineering 4 (7-11), 653-666.

[7] Thwaites, P., 2007. Process control in metallurgical plants. Annual Reviews in Control 31, 221-239.

[8] I. Jovanovic, I Miljanovic, T. Jovanovic, 2015. Soft computing-based modeling of flotation processes - A review, Minerals Engineering 84 (2015) 34-63.

[9] Karray, F., De Silva, C., 2004. Soft Computing and Intelligent Systems Design. Pearson Education, 560 p.

[10] Aldrich, C., Marais, C., Shean, B.J., Cilliers, J.J., 2010. Online monitoring and control of froth flotation systems with machine vision: a review. Int. J. Miner. Process. 96, 1-13.

[11] Bergh, L.G., Yianatos, J.B., Leiva, C.A., 1998. Fuzzy supervisory control of flotation columns. Miner. Eng. 11 (8), 739-748.

[12] B.J. Shean, J.J. Cilliers, 2011, A review of froth flotation control, International Journal of Mineral Processing 100 (2011) 57-71. 